\title{
Diagonally Implicit Runge-Kutta Fourth Order Four-Stage Method for Linear Ordinary Differential Equations with Minimized Error Norm
}

\author{
Nur Izzati Che Jawias ${ }^{1 *}$, Fudziah Ismail ${ }^{1}$, Mohamed Suleiman ${ }^{1}$, Azmi Jaafar ${ }^{2}$ \\ ${ }^{1}$ Department of Mathematics, University Putra Malaysia \\ ${ }^{2}$ Faculty of Computer Science and Information Technology, University Putra Malaysia \\ * Author to whom correspondence should be addressed; E-mail: nurizzatijawias@yahoo.com
}

Received: 25 November 2008, Revised: 12 May 2009

Online Publication: 27 May 2009

http://dx.doi.org/10.11113/mjfas.v5n1.289

\section{ABSTRACT}

We constructed a new fourth order four-stage diagonally implicit Runge-Kutta (DIRK) method which is specially designed for the integrations of linear ordinary differential equations (LODEs). The method is obtained based on the Butcher's error equations. In the derivation, the error norm is minimized so that the free parameters chosen are obtained from the minimized error norm. Row simplifying assumption is also used so that the number of equations for the method can be reduced and simplified. A set of test problems are used to validate the method and numerical results show that the new method is more efficient in terms of accuracy compared to the existing method.

| Runge-Kutta | Linear ordinary differential equations | Error norm |

\section{Introduction}

We consider the numerical integration of linear inhomogeneous systems of ordinary differential equations (ODEs) of the form

$$
y^{\prime}=A y+G(x)
$$

where $A$ is a square matrix whose entries does not depend on $y$ or $x$, and $y$ and $G(x)$ are vectors. Such systems arise in the numerical solution of partial differential equations (PDEs) governing wave and heat phenomena after application of a spatial discretization such as finite-difference method.

Explicit Runge-Kutta method is very popular for simulations of wave equations; see Zingg and Chisholm [8], due to their high accuracy and low memory requirements. 
To derive Runge-Kutta (RK) methods, we need to fulfill certain order equations; see Dormand [3]. These order equations resulted from the derivatives of the function $y^{\prime}=f(x, y)$ itself. If the function is linear then some of the error equations resulted by the nonlinearity in the derivative function can be removed, thus less order equations need to be satisfied, hence a more efficient method in some respect than the classical method can be derived.

In this paper, we construct diagonally implicit Runge-Kutta method specifically for linear ODEs with constant coefficients. We consider the principal terms of the local truncation error to minimize the error norm. Then, the stability aspect of the method is looked into and a few test equations are used to validate the new method.

\section{Materials and Methods}

\section{Derivation of the Method}

In this section, we consider the following scalar ODE

$$
y^{\prime}=f(x, y)
$$

When a general $s$-stage diagonally implicit Runge-Kutta method is applied to the ODE, the following equations are obtained,

$$
y_{n+1}=y_{n}+h \sum_{i=1}^{s} b_{i} k_{i}
$$

where

$$
k_{i}=f\left(x_{n}+c_{i} h, y_{n}+h \sum_{j=1}^{i} a_{i j} k_{j}\right)
$$

We shall always assume that the row-sum condition holds $c_{i}=\sum_{j=1}^{s} a_{i j}$, where $i=1,2 . . s$. According to Dormand [3], the following eight order equations (error equations) are equations needed to be satisfied by fourth order four-stage DIRK method.

Table 2.1: Runge-Kutta order equations for fourth order

\begin{tabular}{|c|l|}
\hline 1. & $\tau_{1}^{(1)}=\sum_{i} b_{i}-1$ \\
\hline 2. & $\tau_{1}^{(2)}=\sum_{i} b_{i} c_{i}-\frac{1}{2}$ \\
\hline 3. & $\tau_{1}^{(3)}=\sum_{i} b_{i} c_{i}^{2}-\frac{1}{3}$ \\
\hline 4. & $\tau_{2}^{(3)}=\sum_{i j} b_{i} a_{i j} c_{j}-\frac{1}{6}$ \\
\hline
\end{tabular}




\begin{tabular}{|r|r|}
\hline 5. & $\tau_{1}^{(4)}=\sum_{i} b_{i} c_{i}^{3}-\frac{1}{4}$ \\
\hline 6. & $\tau_{2}^{(4)}=\sum_{i j} b_{i} c_{i} a_{i j} c_{j}-\frac{1}{8}$ \\
\hline 7. & $\tau_{3}^{(4)}=\sum_{i j} b_{i} a_{i j} c_{j}^{2}-\frac{1}{12}$ \\
\hline 8. & $\tau_{4}^{(4)}=\sum_{i j k} b_{i} a_{i j} a_{j k} c_{k}-\frac{1}{24}$ \\
\hline
\end{tabular}

The restriction to linear ODEs reduces the number of equations which the coefficients of the RK method must satisfy in table 2.1. Zingg and Chisholm [8] have derived new explicit RK methods which are suitable for linear ODEs that are more efficient than the conventional RK methods.

For this new fourth order DIRK method which is suitable for linear ODEs, equation 6 in table 2.1 can be eliminated, as in [8]. This condition is eliminated by exploiting the fact that, for linear ODEs,

$$
\frac{\partial^{2} f}{\partial u^{2}}=\frac{\partial^{2} f}{\partial u \partial t}=0
$$

Using the simplifying assumption:

$$
\sum b_{i} a_{i j}=b_{j}\left(1-c_{j}\right), \quad j=2,3,4
$$

we can removed several equations, i.e. equation 4 and 7 in table 2.1. This makes the new fourth order four-stage DIRK method different from the classical method. So, the number of order equations can be reduced. Thus, the equations needed to be satisfied are:

$$
\begin{aligned}
& j=2 \rightarrow b_{2} \gamma+b_{3} a_{32}+b_{4} a_{42}=b_{2}\left(1-c_{2}\right) \\
& j=3 \rightarrow b_{3} \gamma+b_{4} a_{43}=b_{3}\left(1-c_{3}\right) \\
& j=4 \rightarrow c_{4}=1-\gamma
\end{aligned}
$$

Altogether there are seven equations to be satisfied and we have 10 unknowns. So, we can take three free parameters which are chosen to be $c_{2}, c_{3}$ and $\gamma$. Solving all the related equations, we have all equations in terms of $c_{2}, c_{3}$ and $\gamma$.

Table 2.2: Runge-Kutta order equations for fifth order

\begin{tabular}{|c|c|}
\hline 9. & $\tau_{1}^{(5)}=\frac{1}{24} \sum_{i} b_{i} c_{i}^{4}-\frac{1}{120}$ \\
\hline 10. & $\tau_{2}^{(5)}=\frac{1}{2} \sum_{i j} b_{i} c_{i}^{2} a_{i j} c_{j}-\frac{1}{20}$ \\
\hline
\end{tabular}




\begin{tabular}{|r|r|}
\hline 11. & $\tau_{3}^{(5)}=\frac{1}{2} \sum_{i j k} b_{i} a_{i j} c_{j} a_{i k} c_{k}-\frac{1}{40}$ \\
\hline 12. & $\tau_{4}^{(5)}=\frac{1}{2} \sum_{i j} b_{i} c_{i} a_{i j} c_{j}^{2}-\frac{1}{30}$ \\
\hline 13. & $\tau_{5}^{(5)}=\frac{1}{6} \sum_{i j} b_{i} a_{i j} c_{j}^{3}-\frac{1}{120}$ \\
\hline 14. & $\tau_{6}^{(5)}=\sum_{i j k} b_{i} c_{i} a_{i j} a_{j k} c_{k}-\frac{1}{30}$ \\
\hline 15. & $\tau_{7}^{(5)}=\sum_{i j k} b_{i} a_{i j} c_{j} a_{j k} c_{k}-\frac{1}{40}$ \\
\hline 16. & $\tau_{8}^{(5)}=\frac{1}{2} \sum_{i j k} b_{i} a_{i j} a_{j k} c_{k}^{2}-\frac{1}{120}$ \\
\hline 17. & $\tau_{9}^{(5)}=\sum_{i j k m} b_{i} a_{i j} a_{j k} a_{k m} c_{m}-\frac{1}{120}$ \\
\hline
\end{tabular}

In order to choose the free parameters $c_{2}, c_{3}$ and $\gamma$, the principal terms of the local truncation error must be considered. Using the error function $\varphi_{p}=\sum_{j=1}^{n_{p+1}} \tau_{j}^{(p+1)} F_{j}^{(p+1)}$ and RK error coefficients [3], the principal term for fourth order method is

$$
\varphi_{4}=\sum_{j=1}^{5} \tau_{j}^{(5)} F_{j}^{(5)}
$$

For case of RK suitable for linear ODEs, we only considered $\Gamma_{1}^{(5)}, \Gamma_{5}^{(5)}, \Gamma_{8}^{(5)}$ and $\Gamma_{9}^{(5)}$. Here we can eliminated several equations i.e. $\tau_{2}^{(5)}, \tau_{3}^{(5)}, \tau_{4}^{(5)}, \tau_{6}^{(5)}$ and $\tau_{7}^{(5)}$. The best strategy for practical purposes would be to choose the free RK parameters is to minimize the error norm, see [3];

$$
\mathrm{A}^{(p+1)}=\left\|\tau^{(p+1)}\right\|_{2}=\sqrt{\sum_{j=1}^{n_{p+1}}\left(\tau_{j}^{(p+1)}\right)^{2}}
$$

So we have the principal error norm for this method;

$$
\mathrm{A}^{(5)}=\left\|\tau^{(5)}\right\|_{2}=\sqrt{\left(\tau_{1}^{(5)}\right)^{2}+\left(\tau_{5}^{(5)}\right)^{2}+\left(\tau_{8}^{(5)}\right)^{2}+\left(\tau_{9}^{(5)}\right)^{2}}
$$

where $\tau_{j}^{(5)}$ is the error equations associated with the fifth order method, (in table 2.2). Then we get the principal error norm in terms of $c_{2}, c_{3}$ and $\gamma$. Minimizing the error norm, we obtained $c_{2}=0.36376391115508, c_{3}=0.62453338645147$ and $\gamma=0.091291733465251$. 
Substituting the values of $c_{2}, c_{3}$ and $\gamma$ and solving all the equations we finally get all the coefficients as follows;

$\begin{array}{lllll}0.091291733465251 & & & & \\ 0.27247217768983 & 0.091291733465251 & & \\ 0.18592608940281 & 0.34731556358341 & 0.091291733465251 & \\ 0.23857907019768 & 0.20938627024938 & 0.36945119262243 & 0.091291733465251 \\ 0.22017939484654 & 0.26923249008354 & 0.28860138224069 & 0.22198673282923\end{array}$

Substituting all the parameters into the general form of RK method, we have the new fourth order four-stage DIRK method which is suitable for linear ODEs with minimized error norm,

$$
y_{n+1}=y_{n}+h\left(0.2201 \ldots k_{1}+0.2692 \ldots k_{2}+0.2886 \ldots k_{3}+0.2219 \ldots k_{4}\right)
$$

where

$$
\begin{aligned}
& k_{1}=f\left(x_{n}+0.0912 \ldots h, y_{n}+h\left(0.0912 \ldots k_{1}\right)\right) \\
& k_{2}=f\left(x_{n}+0.3637 \ldots h, y_{n}+h\left(0.2724 \ldots k_{1}+0.0912 \ldots k_{2}\right)\right) \\
& k_{3}=f\left(x_{n}+0.6245 \ldots h, y_{n}+h\left(0.1859 \ldots k_{1}+0.3473 \ldots k_{2}+0.0912 \ldots k_{3}\right)\right) \\
& k_{4}=f\left(x_{n}+0.9087 \ldots h, y_{n}+h\left(0.2385 \ldots k_{1}+0.2093 \ldots k_{2}+0.3694 \ldots k_{3}+0.0912 \ldots k_{4}\right)\right)
\end{aligned}
$$

\section{Stability}

One of the practical criteria for a good method to be useful is that it must have region of absolute stability. When an $s$-stage Runge-Kutta method (equations (2.2a) and (2.2b)) is applied to

$$
\begin{gathered}
y^{\prime}=f(x, y) \\
=\lambda y
\end{gathered}
$$

the following equations is obtained

$$
y_{n+1}=R(h \lambda) y_{n}
$$

with

$$
R(h \lambda)=R(\hat{h})=1+\hat{h} b^{T}(I-\hat{h} A)^{-1} e
$$

where $A$ is ( $m \times m), e$ is $(m \times 1)$ and $R(\hat{h})$ is called the stability polynomial of the method. The stability region is obtained by taking $R(\hat{h})=1=\cos \theta+i \sin \theta$.

From this Butcher's array,

\begin{tabular}{l|l}
$c$ & $a$ \\
\hline & $b^{T}$
\end{tabular}


We can solve for $\hat{h}$ using the Mathematica packaged and get the stability polynomial and also the stability region. The stability polynomial for new fourth order four-stage DIRK method is

$$
\begin{gathered}
1+\hat{h}\left[\frac{0.221987\left(0.209386 \hat{h}+0.0900857 \hat{h}^{2}-0.00996914 \hat{h}^{3}\right)}{1-0.365167 \hat{h}+0.0500051 \hat{h}^{2}-0.00304337 \hat{h}^{3}+0.0000694586 \hat{h}^{4}}+\right. \\
\frac{0.288601\left(0.185926 \hat{h}+0.0606868 \hat{h}^{2}-0.00708974 \hat{h}^{3}\right)}{1-0.365167 \hat{h}+0.0500051 \hat{h}^{2}-0.00304337 \hat{h}^{3}+0.0000694586 \hat{h}^{4}}+ \\
\frac{1 .\left(1-0.273875 \hat{h}+0.0250025 \hat{h}^{2}-0.000760842 \hat{h}^{3}\right)}{1-0.365167 \hat{h}+0.0500051 \hat{h}^{2}-0.00304337 \hat{h}^{3}+0.0000694586 \hat{h}^{4}}+ \\
\frac{0.269232\left(0.272472 \hat{h}-0.0497489 \hat{h}^{2}+0.00227083 \hat{h}^{3}\right.}{1-0.365167 \hat{h}+0.0500051 \hat{h}^{2}-0.00304337 \hat{h}^{3}+0.0000694586 \hat{h}^{4}}+ \\
R(\hat{h})=\frac{0.288601\left(0.347316 \hat{h}-0.0634141 \hat{h}^{2}+0.00289459 \hat{h}^{3}\right)}{1-0.365167 \hat{h}+0.0500051 \hat{h}^{2}-0.00304337 \hat{h}^{3}+0.0000694586 \hat{h}^{4}}+ \\
\frac{0.221987\left(0.369451 \hat{h}-0.0674557 \hat{h}^{2}+0.00307907 \hat{h}^{3}\right.}{1-0.365167 \hat{h}+0.0500051 \hat{h}^{2}-0.00304337 \hat{h}^{3}+0.0000694586 \hat{h}^{4}}+ \\
\left.\frac{0.221987\left(0.238579 \hat{h}+0.082182 \hat{h}^{2}+0.0254717 \hat{h}^{3}\right)}{1-0.365167 \hat{h}+0.0500051 \hat{h}^{2}-0.00304337 \hat{h}^{3}+0.0000694586 \hat{h}^{4}}\right]
\end{gathered}
$$

The stability polynomial is set to zero and solve for $\hat{h}$ which gives the value of $|R(\hat{h})| \leq 1$; this is done by using Mathematica package. The stability region is obtained by tracing the values of $\hat{h}$ and is shown in Figure 2.3. The stability region for new fourth order four-stage DIRK is black in colour.

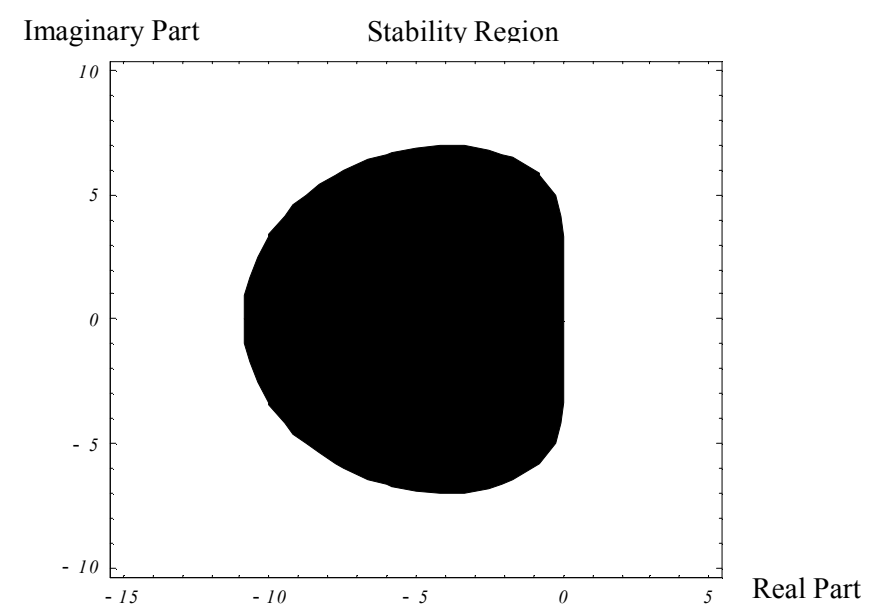

Figure 2.3: The stability region for new $4^{\text {th }}$ order 4 -stage DIRK with minimized error norm 


\section{Results and Discussion}

The following are some of the problems tested. All the problems are linear ODEs.

PROBLEM 1:

$$
\begin{array}{ll}
y^{\prime}(t)=-y & \\
y(t)=e^{-t} & 0 \leq t \leq 1, y(0)=1
\end{array}
$$

Source: Richard L.Burden and J.Douglas Faires (2001)

PROBLEM 2:

$$
\begin{array}{ll}
y^{\prime}(t)=-y \tan t-\frac{1}{\cos t} & 0 \leq t \leq 1, y(0)=1 \\
y(t)=\cos t-\sin t &
\end{array}
$$

Source: J. C. Butcher (2003)

PROBLEM 3:

$$
\begin{aligned}
& y^{\prime}(t)=\frac{2}{t} y+t^{2} e^{t} \\
& y(t)=t^{2}\left(e^{t}-e\right) \\
& 1 \leq t \leq 5, y(1)=0
\end{aligned}
$$

Source: Richard L.Burden and J.Douglas Faires (2001)

The numerical results are tabulated and compared with the existing method and below are the notations used:

- $\mathbf{H}$

- MTHD

- MAXE

- ERK4

- DIRK4 minimized

- SDIRK4,4
Step size used

Method employed

Maximum error $\left|y\left(x_{i}\right)-y_{i}\right|$

Fourth order four-stage explicit RK method (Zingg and Chisholm, 1999 [8])

New fourth order four-stage DIRK method with minimized error norm

Optimal fourth order four-stage singly-DIRK (Ferracina and Spijker,2007 [4])

Table 3.1: Comparison between ERK4 and DIRK4 for solving problem 1

\begin{tabular}{|l|l|c|c|}
\hline & \multicolumn{1}{|c|}{ MTHD } & H & MAXE \\
\hline \multirow{3}{*}{1.} & ERK4 & 0.1 & $3.33241 \mathrm{e}-007$ \\
\cline { 2 - 2 } & DIRK4 minimized & & $1.47717 \mathrm{e}-008$ \\
\cline { 2 - 2 } & SDIRK4,4 & & $1.10407 \mathrm{e}-008$ \\
\cline { 2 - 2 } & ERK4 & \multirow{2}{*}{0.05} & $1.99761 \mathrm{e}-008$ \\
\cline { 2 - 2 } & DIRK4 minimized & & $8.98578 \mathrm{e}-010$ \\
\hline
\end{tabular}




\begin{tabular}{|c|c|c|c|}
\hline & MTHD & $\mathbf{H}$ & MAXE \\
\hline & SDIRK4,4 & & $6.72222 \mathrm{e}-010$ \\
\hline \multirow[t]{3}{*}{3.} & ERK4 & \multirow[t]{3}{*}{0.025} & $1.22274 \mathrm{e}-009$ \\
\hline & DIRK4 minimized & & $5.54087 \mathrm{e}-011$ \\
\hline & SDIRK4,4 & & $4.14714 \mathrm{e}-011$ \\
\hline \multirow[t]{3}{*}{4.} & ERK4 & \multirow[t]{3}{*}{0.01} & $3.09133 \mathrm{e}-011$ \\
\hline & DIRK4 minimized & & $1.40699 \mathrm{e}-012$ \\
\hline & SDIRK4,4 & & $1.05449 \mathrm{e}-012$ \\
\hline \multirow[t]{3}{*}{5.} & ERK4 & \multirow[t]{3}{*}{0.005} & $1.92441 \mathrm{e}-012$ \\
\hline & DIRK4 minimized & & $8.79297 \mathrm{e}-014$ \\
\hline & SDIRK4,4 & & $6.66134 \mathrm{e}-014$ \\
\hline \multirow[t]{3}{*}{6.} & ERK4 & \multirow[t]{3}{*}{0.0025} & $1.16185 \mathrm{e}-013$ \\
\hline & DIRK4 minimized & & $4.66294 \mathrm{e}-015$ \\
\hline & SDIRK4,4 & & $3.77476 \mathrm{e}-015$ \\
\hline \multirow[t]{3}{*}{7.} & ERK4 & \multirow[t]{3}{*}{0.001} & $5.10703 \mathrm{e}-015$ \\
\hline & DIRK4 minimized & & $1.88738 \mathrm{e}-015$ \\
\hline & SDIRK4,4 & & $1.99840 \mathrm{e}-015$ \\
\hline
\end{tabular}

Table 3.2: Comparison between ERK4 and DIRK4 for solving problem 2

\begin{tabular}{|c|c|c|c|}
\hline & MTHD & $\mathbf{H}$ & MAXE \\
\hline 1. & $\begin{array}{l}\text { ERK4 } \\
\text { DIRK4 minimized } \\
\text { SDIRK4,4 }\end{array}$ & 0.1 & $\begin{array}{l}9.88164 \mathrm{e}-006 \\
8.60900 \mathrm{e}-009 \\
1.34285 \mathrm{e}-008\end{array}$ \\
\hline 2. & $\begin{array}{l}\text { ERK4 } \\
\text { DIRK4 minimized } \\
\text { SDIRK4,4 } \\
\end{array}$ & 0.05 & $\begin{array}{l}1.22646 \mathrm{e}-006 \\
1.59223 \mathrm{e}-010 \\
4.30729 \mathrm{e}-010\end{array}$ \\
\hline 3. & $\begin{array}{l}\text { ERK4 } \\
\text { DIRK4 minimized } \\
\text { SDIRK4,4 } \\
\end{array}$ & 0.025 & $\begin{array}{l}1.52419 \mathrm{e}-007 \\
8.36314 \mathrm{e}-012 \\
2.58894 \mathrm{e}-011\end{array}$ \\
\hline 4. & $\begin{array}{l}\text { ERK4 } \\
\text { DIRK4 minimized } \\
\text { SDIRK4,4 }\end{array}$ & 0.01 & $\begin{array}{l}9.72342 \mathrm{e}-009 \\
1.88294 \mathrm{e}-013 \\
6.47565 \mathrm{e}-013\end{array}$ \\
\hline 5. & $\begin{array}{l}\text { ERK4 } \\
\text { DIRK4 minimized } \\
\text { SDIRK4,4 }\end{array}$ & 0.005 & $\begin{array}{l}1.21412 \mathrm{e}-009 \\
1.14908 \mathrm{e}-014 \\
3.98570 \mathrm{e}-014\end{array}$ \\
\hline 6. & $\begin{array}{l}\text { ERK4 } \\
\text { DIRK4 minimized } \\
\text { SDIRK4,4 }\end{array}$ & 0.0025 & $\begin{array}{l}1.51681 \mathrm{e}-010 \\
1.07692 \mathrm{e}-014 \\
1.37113 \mathrm{e}-014\end{array}$ \\
\hline 7. & $\begin{array}{l}\text { ERK4 } \\
\text { DIRK4 minimized } \\
\text { SDIRK4,4 }\end{array}$ & 0.001 & $\begin{array}{l}9.70071 \mathrm{e}-012 \\
4.44089 \mathrm{e}-016 \\
4.44089 \mathrm{e}-016\end{array}$ \\
\hline
\end{tabular}


Table 3.3: Comparison between ERK4 and DIRK4 for solving problem 3

\begin{tabular}{|c|c|c|c|}
\hline & MTHD & H & MAXE \\
\hline \multirow{3}{*}{1.} & ERK4 & \multirow{3}{*}{0.1} & $3.53422 \mathrm{e}-002$ \\
\hline & DIRK4 minimized & & $7.04943 e-005$ \\
\hline & SDIRK4,4 & & $4.98821 \mathrm{e}-006$ \\
\hline \multirow{3}{*}{2.} & ERK4 & \multirow{3}{*}{0.05} & $4.58145 \mathrm{e}-003$ \\
\hline & DIRK4 minimized & & $4.60092 \mathrm{e}-006$ \\
\hline & SDIRK4,4 & & $3.19676 \mathrm{e}-007$ \\
\hline \multirow{3}{*}{3.} & ERK4 & \multirow{3}{*}{0.025} & $5.83708 \mathrm{e}-004$ \\
\hline & DIRK4 minimized & & $2.89703 \mathrm{e}-007$ \\
\hline & SDIRK4,4 & & $1.89380 \mathrm{e}-008$ \\
\hline \multirow{3}{*}{4.} & ERK4 & \multirow{3}{*}{0.01} & $3.77980 \mathrm{e}-005$ \\
\hline & DIRK4 minimized & & $7.51152 \mathrm{e}-009$ \\
\hline & SDIRK4,4 & & $4.04270 \mathrm{e}-010$ \\
\hline \multirow{3}{*}{5.} & ERK4 & \multirow{3}{*}{0.005} & $4.74347 \mathrm{e}-006$ \\
\hline & DIRK4 minimized & & $3.61979 \mathrm{e}-010$ \\
\hline & SDIRK4,4 & & $9.18590 \mathrm{e}-011$ \\
\hline \multirow{3}{*}{6.} & ERK4 & \multirow{3}{*}{0.0025} & $5.93553 \mathrm{e}-007$ \\
\hline & DIRK4 minimized & & $5.08408 \mathrm{e}-010$ \\
\hline & SDIRK4,4 & & $4.77939 \mathrm{e}-010$ \\
\hline \multirow{3}{*}{7.} & ERK4 & \multirow{3}{*}{0.001} & $3.71388 \mathrm{e}-008$ \\
\hline & DIRK4 minimized & & $8.95852 \mathrm{e}-010$ \\
\hline & SDIRK4,4 & & $8.92669 \mathrm{e}-010$ \\
\hline
\end{tabular}

\section{Conclusion}

The new fourth order four-stage DIRK method with minimized error norm has been presented for the integration of linear ODEs. It has a bigger stability region compared to explicit RK method (of the same order), hence more stable. From the numerical results in Table 3.1 to 3.3, we can conclude that the new fourth order four-stage DIRK method which is suitable for linear ODEs performs better in terms of maximum error compared to fourth order four-stage ERK method [8]. This new method is also as good as the optimal fourth order fourstage singly-DIRK [4].

\section{References}

[1] Burden R.L., Faires J.D., Numerical Analysis seventh edition, Wadsworth Group. Brooks/Cole, Thomson Learning, Inc, 2001.

[2] Butcher J.C., Numerical Methods for Ordinary Differential Equation, John Wiley \& Sons Ltd, 2003.

[3] Dormand J.R., Numerical Methods for Differential Equations, Boca Raton, New York, London and Tokya: CRC Press, Inc, 1996.

[4] Ferracina L., Spijker M.N., Strong stability of Singly-Diagonally-Implicit Runge-Kutta methods. Report no MI 2007-11(2007), Mathematical Institute, Leiden University.

[5] Hairer E., Wanner G., Solving Ordinary Differential Equation II, Springer-Verlag Berlin Heidelberg, 1991. 
[6] Lambert J.D., Numerical Methods for Ordinary Differential System, John Wiley \& Sons Ltd, 1991.

[7] Torrence B.F., Torrence E.A.: How to find the stability regions, The Student's Introduction to Mathematica, pp 232-264.

[8] Zingg D.W., Chisholm T.T., Runge-Kutta methods for linear ordinary differential equations, Applied Numerical Mathematics, (1999) 31, 227-238. 